\title{
Gravitational waves in neutrino plasma and NANOGrav signal
}

\author{
Arun Kumar Pandey ${ }^{1,2, a_{\mathbb{D}}}$ \\ ${ }^{1}$ Department of Physics and Astrophysics, University of Delhi, Delhi 110 007, India \\ ${ }^{2}$ School of Fundamental Physics and Mathematical Sciences, Hangzhou Institute for Advanced Study, UCAS, Hangzhou 310024, China
}

Received: 17 March 2021 / Accepted: 25 April 2021 / Published online: 7 May 2021

(C) The Author(s) 2021

\begin{abstract}
The recent finding of the gravitational wave (GW) signal by the NANOGrav collaboration in the $\mathrm{nHZ}$ frequency range has opened up the door for the existence of stochastic GWs. In the present work, we have argued that in a hot dense neutrino asymmetric plasma, GWs could be generated due to the instability caused by the finite difference in the number densities of the different species of the neutrinos. The generated GWs have amplitude and frequency in the sensitivity range of the NANOGrav observation. We have shown that the GWs generated by this mechanism could be one of the possible explanations for the observed NANOGrav signal. We have also discussed generation of GWs in an inhomogeneous cosmological neutrino plasma, where GWs are generated when neutrinos enter a free streaming regime. We show that the generated GWs in an inhomogeneous neutrino plasma cannot explain the observed NANOGrav signal. We have also calculated the lower bound on magnetic fields' strength using the NANOGrav signal and found that to explain the signal, the magnetic fields' strength should have at least value $\sim 10^{-12} \mathrm{G}$ at an Mpc length scale.
\end{abstract}

\section{Introduction}

Albert Einstein first predicted gravitational waves (GWs) in 1916 based on his well-known general theory of relativity. Stochastic gravitational waves are the relic GWs from the early phases of the universe. The detection of stochastic GWs can give us a great insight into the early universe and hence into the high energy physics. These waves can be a direct probe of physics before the recombination era. Despite being so famous and important for several reasons, it could not be directly detected until 2016. When VIRGOLIGO collaborations announced the first detection of the GWs, it originated due to the merger of two black holes of masses 29 and 36 solar masses. This successful detection

\footnotetext{
a e-mails: arunp77@gmail.com; arun_pandey@prl.iitgn.ac.in (corresponding author)
}

of GWs has raised hopes of observing stochastic GWs by susceptible detectors in the future. At present, many ground and space-based experiments are active or are suggested to look for such a signal. The B-mode polarization of Cosmic Microwave Background (CMB) probes GWs by its indirect effects on the CMB $[1,2]$. The ground based experiments for example LIGO (Laser Interferometer Gravitational-Wave Observatory) and advanced VIRGO [3], KAGRA [4] and LIGO-India ${ }^{1}$ have sensitivity at frequency $f \sim 10^{1 \sim 3} \mathrm{~Hz}$. However, the space based GW observations such as LISA [5,6], DECIGO [7], BBO [8] have best sensitivity at frequencies $f \sim \mathrm{mHz}$. The GWs with lower frequencies (of the order of $\sim 10^{-9} \mathrm{~Hz}$ ) are searched for by Pulsar timing arrays (PTA) such as EPTA [9, 10], PPTA [11] and NANOGrav [12].

Recently, one of the PTA experiments, the NANOGrav collaboration, after analyzing the 12.5 years pulsar timing data, reported a signal of the $\mathrm{nHz}$ frequency, which might be strong evidence for the stochastic gravitational waves [12]. Various possible explanations of the observed signal have been given to date. One of the possible sources of the above signal can be of astrophysical origin, for example, mergers of supermassive black-hole binaries [13-15]. Primordial black holes can generate stochastic GWs and are investigated in references [16-21]. String theory-motivated models are also given to describe the observed flat spectrum of $\mathrm{GW}$ in the frequency band [22-24] (for earlier works see references [25-31]). The gravitational waves sourced by magnetic fields and turbulence during the various phases of the early universe are discussed in references [32-35]. Some of the older works based on quantum fluctuation during inflation [36-39], phase transitions [40-42], turbulent phenomenons [43-45], cosmic strings [25] and the magnetic fields [46-48] are the few important sources. In the present work, we have discussed the generation of stochastic GWs above the neutrino decoupling time. Authors of the reference [49] have addressed the generation of GWs when inhomogeneous neutrinos enter the free

\footnotetext{
${ }^{1}$ http://www.gw.iucaa.in/ligo-india/, https://www.ligo-india.in/.
} 
streaming regime at the neutrino decoupling epoch. The nonlinear interaction of neutrino flux with the collective plasma oscillations in a hot dense plasma causes instability and generates turbulence [50,51]. This requires a net lepton number density, $n_{\alpha}(x)=n_{v_{\alpha}}(x)-n_{v_{\bar{\alpha}}}(x)$ (here $\alpha=e, \mu, \tau$, bar denotes the antiparticle) of the neutrinos species before the neutrino decoupling epoch at a length scale $L$, less than the Hubble horizon [52]. The gradient of net lepton number density produces an electric current when the mean free path $\lambda_{v}$ of the neutrinos grows and becomes of the order of $L$ and generates magnetic fields [49,53]. These magnetic fields contribute to an anisotropic energy-momentum tensor and act as a source of gravitational waves. This mechanism works when we consider an inhomogeneous distribution of the lepton number density. However, when a parity-violating interaction of the neutrinos with the leptons is considered, it is shown that the necessity of an inhomogeneous distribution is no longer required [52]. In this case, interactions modify the magneto-hydrodynamic equations [54] and contribute to an additional photon polarization [55]. This additional contribution leads to a new kind of instability of the magnetohydrodynamic modes and generates the magnetic fields at the cost of the homogeneous net lepton number density of neutrinos [47]. The total energy-momentum tensor contains anisotropic stress from the magnetic fields, and they act as a source of the primordial GWs.

We have divided this paper in four sections. In Sect. 2, we have discussed the generation of the magnetic fields and the gravitational waves during the neutrino decoupling epoch in two scenarios: due to parity odd interactions of the leptons with the neutrinos in a neutrino asymmetric plasma, and, due to inhomogeneous distribution of neutrinos at the time of neutrino decoupling epoch. Section 3 contains the discussion of the models discussed in the previous sections in the context of NANOGrav signals and PTA, SKA observations. In the end, we have concluded the result of the present work in Sect. 4. In the present work, we have used Friedman-Robertson-Walker metric for the background space-time

$d s^{2}=-a^{2}(\tau) d \tau^{2}+a^{2}(\tau) \delta_{i j} d x^{i} d x^{j}$,

where the conformal time and the coordinate are represented by $\tau$ and $x^{i}$ respectively and they are connected to the physical coordinate by the relations $d \tau=d t / a$ and $x=x_{\text {phy }} / a$. Above metric is defined in such a way that the scale factor $a(\tau)$ has dimension of length. For a radiation dominated universe $a=1 / T$ and the conformal time $\tau=\left(\frac{90}{8 \pi^{3} g_{\text {eff }}}\right)^{1 / 2} \frac{M_{p l}}{T}, g_{\text {eff }}$ is the effective relativistic degree of freedom at the epoch. We would also like to note here that, we have used natural unit system throughout the present work (for which $\hbar=c=k_{B}=1$ ).

\section{Gravitational waves in a neutrino plasma}

Earlier, various sources have been discussed in the context of the production of stochastic GW background. Of the various possible sources of the GW background, magnetic fields and turbulence in the plasma are the important ones. Since the magnetic fields can contribute to the anisotropic energymomentum tensor and hence GWs, it is natural to ask what are the sources of the magnetic fields. It has been shown that, in a neutrino asymmetric plasma, there is an excess of lepton number density in presence of parity odd interactions of leptons and the neutrino gas. This interaction leads to an additional contribution to the net current proportional to the vorticity in the plasma. This extra term leads to instability and produces magnetic fields at the cost of asymmetry. There are some other works, which show that in the case of inhomogeneous neutrino plasma, magnetic fields could be generated when neutrinos enter the free streaming regime. In the present section, we have first briefly discussed two scenarios under which magnetic fields are generated. Later we have talked about how these magnetic fields could act as a source of GWs. The two possible scenarios are: (i) when parityviolating lepton-neutrino interactions are present, (ii) when an inhomogeneous neutrino plasma enters a free streaming regime. Later a brief description of GW production by individual mechanism is given.

\section{Case-i: Parity violating interactions of the neutrinos}

Parity violation in the context of electron-nucleon interactions $\left(e^{-}+N \rightarrow e^{-}+N\right)$ is well studied. In various experiments, it has been shown that $e^{-}$and $N$ (or in terms of electrons $e^{-}$and quarks $q$ ) are coupled not only by electromagnetic interactions but they are also engaged in neutral weak coupling (for more details see table-1, for Neutral weak interactions in reference [56]). In a hot dense system (for example, in neutron stars), neutrinos behave abnormally and show asymmetry in the particles' number densities over antiparticles. This abnormal behavior of neutrinos is one of the prominent cosmological puzzle. The beyond Standard Model (BSM) framework is one of the possible ways to understand this puzzle. The interaction of the neutrinos $v$ with the leptons $l$ is given by the effective current-current Lagrangian $[56,57]$

$\mathcal{L}_{e f f}=-\sqrt{2} G_{F} \sum_{v_{e}, v_{\mu}, v_{\tau}} J_{v_{\alpha}} J_{l}^{\alpha}$

where $G_{F} \approx 1.17 \times 10^{-5} \mathrm{GeV}^{-2}$ is the Fermi constant and $J_{v \alpha}$ and $J_{l}^{\alpha}$ are defined as axial neutrino currents and vector lepton current. The summation in the above equation is to consider all neutrinos generations. The ensemble average over the neutrino current gives $\left\langle J_{v_{\alpha}}\right\rangle \approx\left(n_{v_{\alpha}}-n_{\bar{v}_{\alpha}}\right)$. This 
additional current $j_{v}^{\beta}$ can be written in terms of polarization tensors $\Sigma^{\alpha \beta} /$ as $j_{v}^{\beta}=\Sigma^{\alpha \beta} A_{\alpha}$. Here the polarization tensor $\Sigma^{\alpha \beta}$ contains three terms, longitudinal, transverse and parity odd term denoted by $\Sigma_{L}^{\alpha \beta}, \Sigma_{T}^{\alpha \beta}$ and $\Sigma_{A}^{\alpha \beta}$ respectively. The odd parity term in the current expression leads to instability in the neutrino plasma, leading to turbulence $[52,55,58]$. The total three current in this case is given by

$j_{v}^{i} \approx \sigma E^{i}-\Sigma_{2} B^{i}-\Sigma_{v} \omega^{i}$

where, $\Sigma_{v}$ is given by $\Sigma_{v} \propto T^{2}$ for $\mu \ll T$ and $\Sigma_{v} \propto$ $\Delta \mu^{2}$ for $\mu \gg T$ (where $\mu$ is the chemical potential). $\Sigma_{2}$ represents the neutrino asymmetry and it is given in reference [47] (see equation 14). In this Eq. (3), the first term represents the ohmic current, the second and third terms come only for the neutrino asymmetric neutrino plasma. Here $\sigma, \Delta \mu$ and $\alpha$ represent the conductivity, asymmetry in the number density of the neutrinos species and the electromagnetic (EM) coupling constant respectively. The symbols $\mathbf{E}, \mathbf{B}$ and $\boldsymbol{\omega}=$ $\nabla \times \mathbf{V}$ are the electric, magnetic and vorticity three vectors respectively (here $\mathbf{V}$ is the velocity vector). Last term in the above equation leads to a term proportional to $\nabla \times \omega$ in the magnetic induction equation, which produces a sufficiently strong magnetic fields. In reference [47], authors have shown that, magnetic fields are generated at the cost of this turbulent kinetic energy. Generation of the magnetic field in the present scenario is given by the following equations

$\frac{\partial E_{B}}{\partial \tau}=\left(-\frac{2 k^{2}}{\sigma}+\frac{\Sigma_{2}}{\sigma}\right) E_{B}+\frac{2 \Sigma_{\nu}^{2} k^{4}}{\sigma^{2}}\left(\tau-\tau_{*}\right) E_{\mathrm{V}}$

where $E_{B}$, and $E_{\mathrm{v}}$ represents the magnetic energy and the turbulent energy density respectively. $\tau_{*}$ is the initial time at which the turbulence is generated and hence the magnetic fields. When sufficiently strong magnetic fields are generated, first term on the right hand side dominates over second term and hence solution can be written as: $E_{B} \propto$ $\operatorname{Exp}\left[-2 \tau k / \sigma\left(k-\Sigma_{2}\right)\right]$. For the wave number $k \leq \Sigma_{2}$, magnetic modes will grow exponentially. However for wave number $k>\Sigma_{2}$, modes will damp.

Case-ii: When inhomogeneous neutrinos enter the free streaming regime

The production of GW, in this case, is briefly described in reference [59]. This mechanism works at a neutrino decoupling epoch. At this epoch, the Hubble horizon was significantly larger than that of EW and QCD phase transitions. This mechanism works when there are net inhomogeneous lepton number density $\left(\Delta n_{\alpha}(x)=n_{v_{\alpha}}(x)-n_{v_{\bar{\alpha}}}(x)\right)$ of one, or more species of the neutrinos before neutrino decoupling epoch at certain length scale $\left(L<H^{-1}\right)$. At the epoch of neutrino decoupling, elastic scattering of the neutrinos to the electrons and positrons creates turbulence, and hence a vortical motion in the plasma. As a result, a gradient of net lepton number density produces an electric current, and hence magnetic fields, when the mean free path $\lambda_{v}$ of the neutrinos grow and become of the order of $L[49,53]$. This can be seen through the following equation [59]

$\frac{\partial \mathcal{K}_{v}}{\partial \tau} \propto \frac{\delta n_{v}}{n_{v}}$

where $\mathcal{K}_{v}$ is the specific momentum flux at a scale $L$. The term on the right hand side produces a non-zero vorticity, i.e $\nabla \times \mathbf{V} \neq 0$ and hence magnetic fields, which can be understood by the Biermann battery equation $\frac{\partial \mathbf{B}}{\partial \tau} \propto(\nabla p \times$ $\nabla \rho$ ) (here $p$ and $\rho$ are the pressure and the number density respectively).

Gravitational wave production

These magnetic fields can contribute to anisotropic stress to the total energy-momentum tensor $T_{i j}$. The transverse traceless (TT) part of energy-momentum tensor $T_{i j}$ can then source the metric perturbation and generates the gravitational waves. The tensor metric perturbations are defined by the metric

$d s^{2}=-a^{2}(\tau) d \tau^{2}+a^{2}(\tau)\left(\delta_{i j}+2 h_{i j}\right) d x^{i} d x^{j}$.

In terms of comoving coordinates and time, the evolution equation of $h_{i j}$ are

$h_{i j}^{\prime \prime}(\tau, k)+2 \mathcal{H} h_{i j}^{\prime}(\tau, k)+k^{2} h_{i j}(\tau, k)=16 \pi G \Pi_{i j}^{T T}(\tau, k)$,

where $\mathcal{H}=\frac{1}{a} \frac{d a}{d \tau}$ is the comoving Hubble parameter. For the radiation dominated and matter dominated, $\mathcal{H}$ is given by $1 / \tau$ and $2 / \tau$ respectively. On the right hand side of Eq. (7), $\Pi_{i j}^{T T}$ is the transverse traceless component of the energy momentum tensor contributed by the magnetic fields, generated in a neutrino plasma and it is given by $\Pi_{i j}^{T T}(\vec{k})=$ $\mathcal{P}_{i k j l}(\vec{k}) T_{k l}(k)$ (here $\mathcal{P}_{i k j l}$ is the projection operator and is given by $\mathcal{P}_{i k j l}(\vec{k})=P_{i k}(\hat{k}) P_{j l}(\hat{k})-\frac{1}{2} P_{i j}(\hat{k}) P_{k l}(\hat{k}), P_{i j}(\hat{k})=$ $\delta_{i j}-\hat{k}_{i} \hat{k}_{j}$ and $\left.\hat{k}=\vec{k} / k\right)$. Here $T_{i j}$ is given as

$T_{i j}=\frac{1}{a^{2}}\left(B_{i} B_{j}-\frac{1}{2} \delta_{i j} B^{2}\right)$.

The energy density of the GW is defined as [60]:

$\rho_{\mathrm{gw}}(\tau, \vec{x})=\frac{1}{32 \pi}\left\langle h_{a b}^{\prime}(\tau, \vec{x}) h^{\prime a b}(\tau, \vec{x})\right\rangle$ 
We define the energy density power spectrum in Fourier space as

$$
\frac{d \Omega_{\mathrm{gw}}}{d \log k}=\frac{k^{3}}{2(2 \pi)^{3} G \rho_{c} a^{2}}\left|h^{\prime}\right|^{2}=\frac{k^{5}}{2(2 \pi)^{3} G \rho_{c} a^{2}}\left|\frac{d h}{d x}\right|^{2}
$$

where $\rho_{c}=3 H_{0}^{2} / 8 \pi G$ is the present day critical density, $h=h_{\mu}^{\mu}$ and $x=k \tau$. The GW energy power spectrum is defined as

$$
\left\langle h_{i j}^{\prime}(\tau, \vec{p}) h_{i j}^{\prime *}(\tau, \vec{q})\right\rangle=(2 \pi)^{3} \delta(\vec{p}-\vec{q})\left|h^{\prime}(\tau, p)\right|^{2} .
$$

The generated GW will decay only through the expansion of the universe and scales as $1 / a^{4}$, when the considered wavelength is inside the horizon (i.e. $x \gg 1$ ). At such a scale, $\mathrm{GW}$ energy density power spectrum at present is given by [47]

$$
\left.\frac{d \Omega_{\mathrm{gw}}}{d \ln k}\right|_{0}=\left.\frac{\Omega_{\mathrm{GW}}}{d \ln k}\right|_{*}\left(\frac{g_{s_{0}}}{g_{s_{*}}}\right)^{4 / 3}\left(\frac{T_{0}}{T_{*}}\right)^{4} \frac{\rho_{c, *}}{\rho_{c, 0}},
$$

where $g_{s}$ is the degree of freedom, $T$ is the temperature, subscript ' 0 ' and ' $*$ ' shows that values at present-day and the generation time respectively. To find this expression, we need to find the solution of Eq. (7). It has been shown in reference [47] that, in the case of neutrinos in a hot dense plasma, generated GW energy density power spectrum at present is given by

$$
\begin{aligned}
\left.\frac{d \Omega_{\mathrm{gw}}}{d \ln k}\right|_{0}= & \frac{64 \pi M_{\mathrm{st}}^{2} k^{3}}{9(2 \pi)^{6}}\left(\frac{g_{s 0}}{g_{s}}\right)^{4 / 3}\left(\frac{T_{0}}{T_{*}}\right)^{2} \\
& \times\left(\frac{T_{0}}{H_{0}}\right)^{2}\left[\left.\ln \left(x_{*}\right) \frac{\partial}{\partial \tau}\left(\frac{\sin x}{x}\right)\right|_{\tau=\tau_{*}}\right]^{2} f(k),
\end{aligned}
$$

where $M_{\mathrm{st}}=\left(\frac{90}{8 \pi^{3} g_{\text {geff }}}\right)^{1 / 2} M_{\mathrm{pl}}$. Here $f(k)$ is given by the relation

$$
\begin{aligned}
f(k)= & \frac{1}{4} \frac{1}{(4 \pi)^{2}} \int d^{3} p\left[\left(1+\gamma^{2}\right)\left(1+\beta^{2}\right) S(p) S(k-p)\right. \\
& +4 \gamma \beta A(p) A(k-p)] .
\end{aligned}
$$

In above equations, $\gamma=\hat{\mathbf{k}} \cdot \hat{\mathbf{p}}$ and $\beta=\hat{\mathbf{k}} \cdot(\widehat{\mathbf{k}-\mathbf{p}})$. The symmetric part $S(k)$ and antisymmetric part $A(k)$ of the two point correlator of magnetic fields, i.e. $\left\langle\mathrm{B}_{i}(\vec{k}) \mathrm{B}_{j}\left(\vec{k}^{\prime}\right)\right\rangle$ are related to the magnetic energy and helical magnetic energy density of the magnetic fields and they are defined as: $\Omega_{B}(k)=2 \pi k^{3} S(k) / \rho_{c}$ and $\Omega_{H}(k)=2 \pi k^{2} A(k) / \rho_{c}$. From the Eq. (13) it is thus clear that the energy density spectrum of GWs are mainly governed by the behavior of $f(k)$ at different scales, defined in Eq. (14).

\section{Result and discussion}

In this section, we interpret the NANOGrav signal as a primordial GWs background generated by the magnetic fields in a hot dense parity odd neutrino plasma. Later we discuss the statistical properties of the GWs induced in a neutrino asymmetric plasma and calculate the favored slope in current context. We have also calculated the strength of the magnetic fields from the observed NANOGrav signal. It is important to note here that, we have not considered any back reaction of GWs on the neutrino plasma. The back reaction has important consequences on neutrino flavor oscillations and it has been shown that due to the interactions fluxes at the detectors are not same $[61,62]$. Since we are not interested in the study of flavor oscillations and mass measurement of neutrinos, we ignore any such effects in our present work.

\subsection{NANOGrav signal and neutrino induced GW signature}

A highly magnetized and rapidly rotating neutron star that emits a beam of electromagnetic radiation along its magnetic axis is known as a pulsar. An observer observes these radiations fairly precisely. The arrival time of pulses is extremely regular and can be predicted accurately over a long period of time. When a GW passes between the observer and the pulsar, it changes the time of arrival pulses proportional to the amplitude of the GW. By monitoring the fluctuations in the time of arrival of the radio pulses, international pulsar timing array (IPTA) plan to probe the stochastic GW background. The observation time sets the PTA experiments maximum sensitivity (i.e. lowest detectable frequency). It means that sensitivity of the detectors are inverse of the time span of the data set (for example, a frequency of $\mathrm{nHz}$ order requires a datasets spanning $\sim 10$ years). One of the PTA experiments, the NANOGrav collaboration, 12.5 years of data is sensitivity to GW frequencies between approximately $2.5 \mathrm{nHZ}$ to $1 \mu \mathrm{HZ}$ [12]. NANOGrav observes a strong preference of a stochastic common-spectrum process, modeled as a power law, i.e. $\Omega_{\mathrm{GW}} \propto f^{\zeta}$ in a narrow frequency range around $f \sim 5.5 \mathrm{nHz}$. The fitted spectrum has amplitude $\Omega_{\mathrm{GW}}(f=5.5 \mathrm{nHz}) \in\left(3 \times 10^{-10}-2 \times 10^{-9}\right)$ and exponent $\zeta \in(-1.5,0.5)$ at $1 \sigma$ confidence level. Of the various possible sources of the stochastic GW background at such a frequencies is inspiraling supermassive black hole (SMBH) with an exponent $\zeta \sim-2 / 3$.

Figure 1 shows the plots of the present study (red-dashed line) along with the sensitivities of the NANOGrav (lightgreen shaded region), IPTA (light-orange) and the SKA (light-blue) collaborations. In the present study, we show that the generated magnetic fields in a hot dense neutrino plasma act as a source of the GWs (see Eqs. (7) and (8)). In Fig. 1, it is clear that the amplitude and the frequency of the GWs (red-dashed line) generated by magnetic fields at tempera- 
ture $T \sim 10^{4} \mathrm{GeV}$ in a neutrino asymmetric plasma lie in the allowed range of the NANOGrav experiment at a frequency ( $f \sim 10^{-8} \mathrm{~Hz}$ ). At lower temperatures, the spectrums' peak shifted towards the lower frequency, but amplitudes are so small that they are out of reach of the NANOGrav sensitivity. In a previous work [47], it has been shown that the GWs originated at lower temperatures $\left(T<10^{4} \mathrm{GeV}\right)$ can be detected in the IPTA and SKA observations. Therefore we, believe that one of the possible explanations for the detected signal by NANOGrav collaborations is the GWs generated in a neutrino plasma above neutrino decoupling, where parity odd interactions of the neutrinos with the leptons are dominant. In this case, we need not have to consider the inhomogeneous distribution of the neutrinos in the plasma. In the case of inhomogeneous neutrino density, the produced GWs have energy density $\Omega_{\mathrm{gw}} h^{2} \simeq 9.5 \times 10^{-7} \lambda_{0}^{3 / 4} v^{6}\left(x_{*}\right)$ and frequency $f_{0} \simeq 10^{-7} \lambda_{0}^{-1}$ (here $\lambda_{0}$ is the comoving wavelength, $f_{0}$ is corresponding frequency and $v$ represents the velocity field) [49]. We have found that, although amplitude $\left(\sim 10^{-10}\right)$ lies in the NANOGrav lower limit, frequency ( $f \sim 10^{-6} \mathrm{~Hz}$ ) is beyond the reach of the experiment. Therefore, the produced GWs can not explain the observed GWs by the NANOGrav collaboration. Instead, these GWs could be detected by space-based experiments (eLISA).

Earlier, various lepton asymmetric and phase transition models were given to explain the observed magnetic fields and hence GWs. For example, in reference [46] (for similar work based on baryogenesis and leptogenesis, see references $[63,64])$, it has been shown that at a temperature above EW phase transitions ( $\mathrm{T} \geq 100 \mathrm{GeV}$ ), due to the chiral asymmetry of the electrons, strong magnetic fields are generated and these fields later act as a source for the GWs. However, the frequency of the GWs generated due to the magnetic fields at temperature $\mathrm{T} \sim 10^{4} \mathrm{GeV}$ in a chiral asymmetric fluid is of the order of $\sim 10^{-1} \mathrm{~Hz}$. Therefore, these GWs can not explain the observed NANOGrav signal. In EW phase transition models, for example in reference [40], produced GWs can not explain NANOGrav signals as the frequency of these GWs are of few $\mathrm{mHz}$. In these models, the source of the gravity waves is colliding bubbles and hydrodynamic turbulence at the cosmological phase transitions. In a more recent work based on the QCD phase transition [32] (see also [35]), authors have described the observed NANOGrav signal as a product of the magnetohydrodynamic (MHD) turbulence at the phase transition. Gravitational-wave signatures resulting from the strong first-order phase transition due to the presence of the Higgs doublet have been discussed in the reference [65]. However, the frequency $\left(10^{-2}-10^{-3} \mathrm{~Hz}\right)$ of these GWs is much higher than the frequency of the observed GW by NANOGrav collaboration and hence again cannot explain the NANOGrav signal. A similar situation of lepton number asymmetry can arise in the case of Quark-Gluon
Plasma (QGP) at temperature $T \sim 100 \mathrm{MeV}$. In the case of the merger of neutron stars in a binary system, the observational signature of the gravitational waves is discussed in reference [66]. It is believed that quarks and gluons are the major constituents at such a high temperature in the core of the neutron stars. Such mergers represent potential sites for a phase transition from a confined hadronic matter to deconfined quark matter.In a fully general-hydrodynamic simulation, it is shown that a similar GWs signature from the merger of neutron stars GW170817 (LIGO collaboration [66]) can be obtained in the case of QGP phase transition [67]. The obtained frequency of these GWs are in the sensitivity range of LIGO and hence cannot explain the NANOGrav signal. Therefore, we believe that of all possible models based on the phase transitions, chiral asymmetric models above EW phase transitions, neutrino asymmetric models of generation of GWs is one of the suitable models to explain the NANOGrav signal.

\subsection{Statistical properties and power law background}

From various theoretical magnetohydrodynamic models, it is expected that the power spectrum of the stochastic GW background to be a broken power-law $f^{\beta}$. In a super Horizon frequency range, where frequency $f<f_{H}=a H$, the slope $\beta=3$. However, around source frequency, $0<\beta \leq 3$ [68] and at frequencies $f \geq f_{*}$ (here $f_{*}$ is the source frequency), the slope $\beta<0$ [69]. Normally slope $\beta$ depends on the initial conditions of magnetic fields, type of MHD turbulence and its temporal evolution and the decorrelation time. The characteristic strain spectrum $h_{c}(f)$ describes the GW background in the experiments and it is normally expressed as a function of dimensionless amplitude $A_{\mathrm{CP}}$ at a reference frequency $f_{\mathrm{yr}}=1 / \mathrm{yr} \sim 10^{-8} \mathrm{~Hz}$ (inverse of time in year) $[70,71]$

$h_{c}(f)=A_{\mathrm{CP}}\left(\frac{f}{f_{\mathrm{yr}}}\right)^{\alpha_{\mathrm{CP}}}$,

where the subscript " $\mathrm{CP}$ " represents the common-spectrum (CP) process (common to the observed pulsars). The spectral index $\alpha_{\mathrm{CP}}$ is the slope of the GW strain and depends on the source of the GW background. The choice of the reference frequency $f_{\mathrm{yr}}$ is a arbitrary and it doesn't affect the detectability of the GW by the detector.

The scaling of the $\Omega_{\mathrm{GW}}$ is interpreted as the frequency dependence at the peak of the GW power spectrum. To understand the observed stochastic GW background on a detector, we need to compare the theoretical model to the fitted power law given in Eq. (15). In a transverse traceless gauge

$$
\left\langle h_{i j}^{\mathrm{TT}}(t) h_{i j}^{\mathrm{TT}}(t)\right\rangle=2 \int_{f=\infty}^{f=f} d \log f h_{c}^{2}(f),
$$




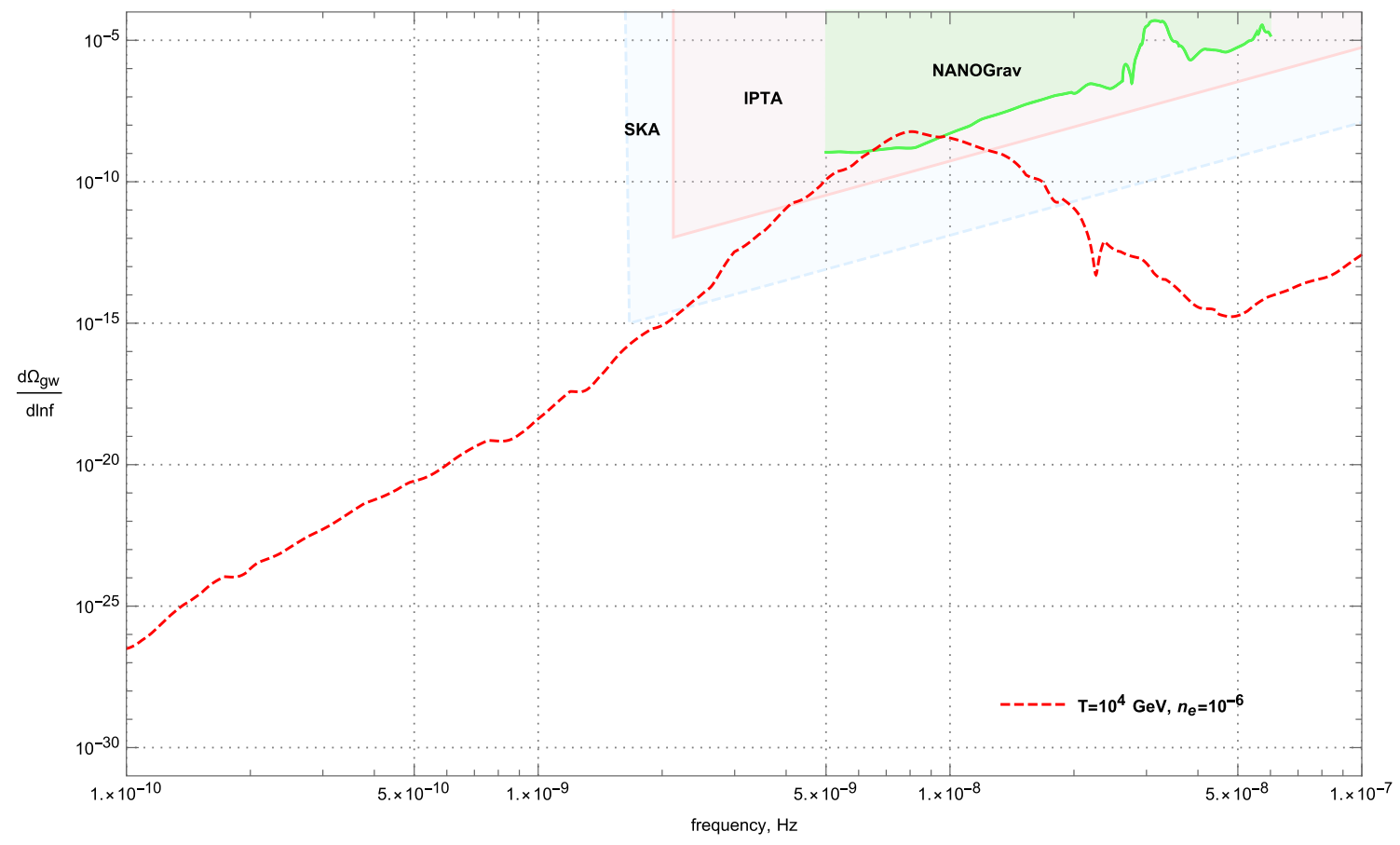

Fig. 1 GW spectrum at present: The light green shaded area is the sensitivity range of the NANOGrav observation. The light orange range represents the sensitivity range of the IPTA experiment and the light blue shaded range is the sensitivity of the SKA. The dashed red lines show the GW power spectrum for the GWs generated by the magnetic fields, which have sourced by the magnetic fields, produced in a hot dense neutrino plasma (electron density of $10^{-6}$ ). Here we have chosen the plasma electrical conductivity of $\sigma \sim 100$ where the angular brackets denote the ensemble average for the stochastic GW background. The factor 2 on the right hand side in above equation is motivated by the fact that, in an unpolarized background, the left hand side is made up of two contributions, $\left\langle h_{+}^{*} h_{+}\right\rangle$and $\left\langle h_{-}^{*} h_{-}\right\rangle$. The GW power spectrum observed today is given by

$\left.\frac{d \Omega_{\mathrm{GW}}}{d \ln f}\right|_{0}=\frac{1}{\rho_{c, 0}} \frac{d \rho_{\mathrm{GW}}(f)}{d \log f}=\frac{\pi c^{2}}{4 G \rho_{c}} f^{2} h_{c}^{2}(f)$.

Therefore, power spectrum of the GW today, using Eq. (10) can be expressed as

$$
\left.\frac{d \Omega_{\mathrm{GW}}}{d \ln f}\right|_{0}=\frac{\pi c^{2}}{4 G \rho_{c, 0}} f_{\mathrm{yr}}^{2} A_{\mathrm{CP}}^{2}\left(\frac{f}{f_{\mathrm{yr}}}\right)^{5-2 \gamma_{\mathrm{CP}}}
$$

where $\gamma_{\mathrm{CP}}$ and $\alpha_{\mathrm{CP}}$ are related to each other by $\gamma_{\mathrm{CP}}=$ $\left(3-2 \alpha_{\mathrm{CP}}\right)$. The reported signal of stochastic background of GW using 12.5 years of timing residual data set has frequency components in the frequency range of $\left[2.5 \times 10^{-9}\right.$ $\left.7.0 \times 10^{-8}\right] \mathrm{Hz}$ [12]. The posteriors for a common spectrum process in the NANOGrav 12.5 year data were recovered for free-spectrum, broken power law, 5-frequency power law, and 30-frequency power law. In the NANOGrav collaboration paper [12], the time residual power spectrum density has been modeled as a power law and broken power law in frequency. For such a spectrum, a 68 and $95 \%$ confidence regimes are obtained for the amplitude and the spectral index using the lowest 5 frequency components. In our work, it is the broken power law, which is more relevant than the prior. For our case, we have compared two equations, (10) and (18):

$$
\begin{aligned}
A_{\mathrm{CP}}^{2}= & \frac{128 M_{\mathrm{st}}^{4} G \rho_{c, 0} f_{\mathrm{yr}}^{3}}{81 \pi H_{0}^{2}}\left(\frac{g_{s 0}}{g_{s} *}\right)^{4 / 3}\left(\frac{T_{0}}{T_{*}}\right)^{4} \\
& \times\left[\log \left(\frac{2 \pi f_{*} M_{\mathrm{st}}}{T_{*}^{2}}\right)\right]^{2} B_{0}^{4}, \\
\alpha_{\mathrm{CP}}= & -\frac{1}{2} .
\end{aligned}
$$

Here we have compared the two equations at the peak of the GW spectrum for the superhorizon GW modes after considering that near peak, $f(k) \approx B_{0}^{4}$ (where $B_{0}$ is the present day large scale magnetic field strength). Therefore, for a $\mathrm{GW}$ produced in a neutrino asymmetric plasma above the neutrino decoupling epoch, $\alpha=-1 / 2$ (which correspondence to $\left.\gamma_{\mathrm{CP}}=4\right)$ at the peak of the GW power spectrum $[12,32,69,72]$. The calculated slope is well within the $2 \sigma$ bounds obtained for the slope of the broken power law spectrum of the GWs by the NANOGrav collaborations (see figure 1 of $A_{\mathrm{CP}}-\alpha_{\mathrm{CP}}$ posterior distribution plot of reference [12]). One of the important difference of our model of GW production with that of the models based on SMBHs is the slop of the GW spectrum. In the case of SMBHs, it has a 
value of $-2 / 3$ and in our case, it is $-1 / 2$ around the peak of GW evolution.

\subsection{Favored strength of magnetic fields}

From Eq. (8), we can write the transverse traceless part of the total energy-momentum tensor as: $\Pi^{T T} \sim B^{2} / 2$. In equilibrium, tensor perturbations $h \sim 16 \pi G \Pi^{T T} / k^{2} \simeq$ $8 \pi G B^{2} / a k^{2}$. The comoving energy density spectrum per logarithmic scale, in terms of the magnetic field, can be expressed as $\Omega_{\mathrm{gw}}=k^{2} h^{2} /\left(32 \pi G \rho_{c}\right) \propto f(k)$. It is thus apparent that the spectrum of the GW depends on the nature of the function $f(k)$ and hence on the magnetic field origin method. The strength of the magnetic fields, induced in a neutrino asymmetric plasma in a hot dense plasma above the time of neutrino decoupling, could be constrained by considering the fact that the generated GWs will have amplitude at least in the NANOGrav sensitivity range. Which means that $\Omega_{\mathrm{GW}, 0} \geq k^{2} h_{\min }^{2} /\left(32 \pi G \rho_{c}\right)$ and hence

$B_{\min }^{2}=\sqrt{\frac{\Omega_{\mathrm{GW}, 0} \rho_{c}}{2 G}\left(\frac{2 \pi f}{c}\right)^{2}}$.

It is thus obvious that to explain NANOGrav signal at frequency $f \sim 10^{-8} \mathrm{~Hz}$, strength of the magnetic fields generated at temperature $\mathrm{T} \sim 10^{4} \mathrm{GeV}$, should have a minimum value of the order of $1.3 \times 10^{-12} \mathrm{G}$ at a coherence scale of $\sim 1 \mathrm{Mpc}$ length scale at present.

\section{Conclusion}

In conclusion, we have found that the GWs produced in a homogeneous neutrino plasma can have amplitude and the frequency in the sensitivity range of the NANOGrav experiment if these GWs were generated much above the neutrino decoupling epoch and there are parity odd interactions between the neutrinos and the leptons. However, GWs generated in an inhomogeneous neutrino plasma, sourced by the magnetic fields, cannot explain the observed NANOGrav signal.It is thus clear from the present work that apart from the well-studied mechanism of the generation of the primordial GWs by various phase transitions, inflation, or some turbulent phenomena in the early universe, the proposed mechanism in the present study is also one of the possible explanations for the observed signal.

Acknowledgements A. K. P. acknowledge the facilities at I.C.A.R.D, Department of Physics and Astrophysics, University of Delhi, Delhi, India. A.K.P. is financially supported by the Dr. D. S. Kothari PostDoctoral Fellowship provided by UGC, Gov. of India, under the Grant No. DSKPDF Ref. No. F.4-2/2006 (BSR)/PH /18-19/0070. A. K. P would also likes to thanks Prof. T. R. Seshadri and Dr. Sampurn Anand for the useful discussions and the comments during the work.
Data Availability Statement This manuscript has no associated data or the data will not be deposited. [Authors' comment: Data sharing not applicable to this article as this work is an outcome of a theoretical construct. The external data used in the study is publicly available at the NANOGrav collaboration website and it has been properly cited in the article.]

Open Access This article is licensed under a Creative Commons Attribution 4.0 International License, which permits use, sharing, adaptation, distribution and reproduction in any medium or format, as long as you give appropriate credit to the original author(s) and the source, provide a link to the Creative Commons licence, and indicate if changes were made. The images or other third party material in this article are included in the article's Creative Commons licence, unless indicated otherwise in a credit line to the material. If material is not included in the article's Creative Commons licence and your intended use is not permitted by statutory regulation or exceeds the permitted use, you will need to obtain permission directly from the copyright holder. To view a copy of this licence, visit http://creativecomm ons.org/licenses/by/4.0/.

Funded by $\mathrm{SCOAP}^{3}$.

\section{References}

1. M. Zaldarriaga, U. Seljak, Phys. Rev. D 55, 1830 (1997). https:// doi.org/10.1103/PhysRevD.55.1830

2. M. Kamionkowski, A. Kosowsky, A. Stebbins, Phys. Rev. D 55, 7368 (1997). https://doi.org/10.1103/PhysRevD.55.7368

3. F. Acernese, J. Phys. Conf. Ser. 610(1), 012014 (2015). https://doi. org/10.1088/1742-6596/610/1/012014

4. K. Somiya, Class. Quantum Gravity 29(12), 124007 (2012). https:// doi.org/10.1088/0264-9381/29/12/124007

5. P. Amaro-Seoane et al., GW Notes 6, 4 (2013)

6. P. Amaro-Seoane, et al., (2017), arXiv:1702.00786 [astro-ph.IM]

7. S. Kawamura et al., Class. Quantum Gravity 28, 094011 (2011). https://doi.org/10.1088/0264-9381/28/9/094011

8. J. Crowder, N.J. Cornish, Phys. Rev. D 72, 083005 (2005). https:// doi.org/10.1103/PhysRevD.72.083005

9. L. Lentati et al., Mon. Not. R. Astron. Soc. 453(3), 2576 (2015). https://doi.org/10.1093/mnras/stv1538

10. Z. Arzoumanian, P.T. Baker, A. Brazier, S. Burke-Spolaor, S.J. Chamberlin, S. Chatterjee, B. Christy, J.M. Cordes, N.J. Cornish, F. Crawford et al., Astrophys. J. 859(1), 47 (2018). https://doi.org/ 10.3847/1538-4357/aabd3b

11. J.P.W. Verbiest, L. Lentati, G. Hobbs, R. van Haasteren, P.B. Demorest, G.H. Janssen, J.B. Wang, G. Desvignes, R.N. Caballero, M.J. Keith et al., Mon. Not. R. Astron. Soc. 458(2), 1267-1288 (2016). https://doi.org/10.1093/mnras/stw347

12. Z. Arzoumanian et al., Astrophys. J. Lett. 905(2), L34 (2020). https://doi.org/10.3847/2041-8213/abd401

13. M. Rajagopal, R.W. Romani, Astrophys. J. 446, 543 (1995). https:// doi.org/10.1086/175813

14. A.H. Jaffe, D.C. Backer, Astrophys. J. 583, 616 (2003). https://doi. org/10.1086/345443

15. J.B. Wyithe, A. Loeb, Astrophys. J. 590, 691 (2003). https://doi. org $/ 10.1086 / 375187$

16. V. Vaskonen, H. Veermäe, Phys. Rev. Lett. 126, 051303 (2021). https://doi.org/10.1103/PhysRevLett.126.051303

17. S. Bhattacharya, S. Mohanty, P. Parashari, Phys. Rev. D 103, 063532 (2021). https://doi.org/10.1103/PhysRevD.103.063532

18. V. De Luca, G. Franciolini, A. Riotto, Phys. Rev. Lett. 126, 041303 (2021). https://doi.org/10.1103/PhysRevLett.126.041303

19. Q. Ding, X. Tong, Y. Wang, Astrophys. J. 908(1), 78 (2021). https:// doi.org/10.3847/1538-4357/abd803 
20. C. Xin, C.M.F. Mingarelli, J.S. Hazboun, arXiv:2009.11865 [astroph.GA]

21. R.G. Cai, Z.K. Guo, J. Liu, L. Liu, X.Y. Yang, JCAP 06, 013 (2020). https://doi.org/10.1088/1475-7516/2020/06/013

22. J. Ellis, M. Lewicki, Phys. Rev. Lett. 126, 041304 (2021). https:// doi.org/10.1103/PhysRevLett.126.041304

23. S. Blasi, V. Brdar, K. Schmitz, Phys. Rev. Lett. 126, 041305 (2021). https://doi.org/10.1103/PhysRevLett.126.041305

24. W. Buchmuller, V. Domcke, K. Schmitz, Phys. Lett. B 811, 135914 (2020). https://doi.org/10.1016/j.physletb.2020.135914

25. A. Vilenkin, Phys. Lett. B 107, 47 (1981). https://doi.org/10.1016/ 0370-2693(81)91144-8

26. T. Vachaspati, A. Vilenkin, Phys. Rev. D 31, 3052 (1985). https:// doi.org/10.1103/PhysRevD.31.3052

27. P. Binetruy, A. Bohe, C. Caprini, J.F. Dufaux, JCAP 1206, 027 (2012). https://doi.org/10.1088/1475-7516/2012/06/027

28. M. Gasperini, M. Giovannini, G. Veneziano, Phys. Rev. Lett. 75, 3796 (1995). https://doi.org/10.1103/PhysRevLett.75.3796

29. D. Lemoine, M. Lemoine, Phys. Rev. D 52, 1955 (1995). https:// doi.org/10.1103/PhysRevD.52.1955

30. C. Ringeval, M. Sakellariadou, F. Bouchet, JCAP 02, 023 (2007). https://doi.org/10.1088/1475-7516/2007/02/023

31. X. Siemens, V. Mandic, J. Creighton, Phys. Rev. Lett. 98, 111101 (2007). https://doi.org/10.1103/PhysRevLett.98.111101

32. A. Neronov, A. Roper Pol, C. Caprini, D. Semikoz, Phys. Rev. D 103(4), 041302 (2021). https://doi.org/10.1103/PhysRevD.103. L041302

33. S. Vagnozzi, Mon. Not. R. Astron. Soc. 502, L11 (2021). https:// doi.org/10.1093/mnrasl/slaa203

34. Y. Nakai, M. Suzuki, F. Takahashi, M. Yamada, Phys. Lett. B 816, 136238 (2021). https://doi.org/10.1016/j.physletb.2021.136238

35. C. Caprini, R. Durrer, X. Siemens, Phys. Rev. D 82, 063511 (2010). https://doi.org/10.1103/PhysRevD.82.063511

36. V.A. Rubakov, M.V. Sazhin, A.V. Veryaskin, Phys. Lett. 115B, 189 (1982). https://doi.org/10.1016/0370-2693(82)90641-4

37. M. Giovannini, Phys. Rev. D 60, 123511 (1999). https://doi.org/ 10.1103/PhysRevD.60.123511

38. R. Sharma, K. Subramanian, T. Seshadri, Phys. Rev. D 101(10), 103526 (2020). https://doi.org/10.1103/PhysRevD.101.103526

39. R. Sharma (2021). arXiv:2102.09358 [astro-ph.CO]

40. M. Kamionkowski, A. Kosowsky, M.S. Turner, Phys. Rev. D 49, 2837 (1994). https://doi.org/10.1103/PhysRevD.49.2837

41. A. Kosowsky, M.S. Turner, R. Watkins, Phys. Rev. D 45, 4514 (1992). https://doi.org/10.1103/PhysRevD.45.4514

42. E. Witten, Phys. Rev. D 30, 272 (1984). https://doi.org/10.1103/ PhysRevD.30.272

43. A. Kosowsky, A. Mack, T. Kahniashvili, Phys. Rev. D 66, 024030 (2002). https://doi.org/10.1103/PhysRevD.66.024030

44. A.D. Dolgov, D. Grasso, A. Nicolis, Phys. Rev. D 66, 103505 (2002). https://doi.org/10.1103/PhysRevD.66.103505

45. T. Kahniashvili, G. Gogoberidze, B. Ratra, Phys. Rev. Lett. 95, 151301 (2005). https://doi.org/10.1103/PhysRevLett.95.151301

46. S. Anand, J.R. Bhatt, A.K. Pandey, Eur. Phys. J. C 79(2), 119 (2019). https://doi.org/10.1140/epjc/s10052-019-6619-5

47. A.K. Pandey, P.K. Natwariya, J.R. Bhatt, Phys. Rev. D 101(2), 023531 (2020). https://doi.org/10.1103/PhysRevD.101.023531
48. T. Fujita, K. Kamada, Y. Nakai, Phys. Rev. D 102(10), 103501 (2020). https://doi.org/10.1103/PhysRevD.102.103501

49. A.D. Dolgov, D. Grasso, Phys. Rev. Lett. 88, 011301 (2002). https://doi.org/10.1103/PhysRevLett.88.011301

50. R. Bingham, J. Dawson, J. Su, H. Bethe, Phys. Lett. A 193(3), 279 (1994). https://doi.org/10.1016/0375-9601(94)90597-5

51. V.N. Tsytovich, R. Bingham, J.M. Dawson, H.A. Bethe, Astropart. Phys. 8, 297 (1998). https://doi.org/10.1016/ S0927-6505(97)00044-3

52. M. Dvornikov, V.B. Semikoz, JCAP 1405, 002 (2014). https://doi. org/10.1088/1475-7516/2014/05/002

53. S. Boldyrev, F. Cattaneo, Phys. Rev. Lett. 92, 14 (2004). https:// doi.org/10.1103/physrevlett.92.144501

54. F. Haas, K.A. Pascoal, Phys. Plasmas 012104, 23 (2017). https:// doi.org/10.1063/1.4997187

55. J.R. Bhatt, M. George, Int. J. Mod. Phys. D 26(06), 1750052 (2016). https://doi.org/10.1142/S0218271817500523

56. E. Commins, P. Bucksbaum, Ann. Rev. Nucl. Part. Sci. 30, 1 (1980) https://doi.org/10.1146/annurev.ns.30.120180.000245

57. C. Giunti, C.W. Kim (Oxford University Press, Oxford, 2007) https://doi.org/10.1093/acprof:oso/9780198508717.001.0001

58. Y. Akamatsu, N. Yamamoto, Phys. Rev. Lett. 111, 052002 (2013). https://doi.org/10.1103/PhysRevLett.111.052002

59. A.D. Dolgov, D. Grasso, Phys. Rev. Lett. 88, 011301 (2001). https://doi.org/10.1103/PhysRevLett.88.011301

60. R.A. Isaacson, Phys. Rev. 166, 1263 (1968). https://doi.org/10. 1103/PhysRev.166.1263

61. M. Dvornikov, J. Cosmol. Astropart. Phys. 2020(12), 022 (2020). https://doi.org/10.1088/1475-7516/2020/12/022

62. M. Dvornikov, Phys. Rev. D 100, 9 (2019). https://doi.org/10.1103/ physrevd.100.096014

63. A. Beniwal, M. Lewicki, M. White, A.G. Williams, JHEP 02, 183 (2019). https://doi.org/10.1007/JHEP02(2019)183

64. K.P. Xie, L. Bian, Y. Wu, JHEP 12, 047 (2020). https://doi.org/10. 1007/JHEP12(2020)047

65. B. Barman, A. Dutta Banik, A. Paul, Phys. Rev. D 101(5), 055028 (2020). https://doi.org/10.1103/PhysRevD.101.055028

66. B. Abbott et al., Phys. Rev. Lett. 119, 161101 (2017). https://doi. org/10.1103/PhysRevLett.119.161101

67. L.R. Weih, M. Hanauske, L. Rezzolla, Phys. Rev. Lett. 124, 171103 (2020). https://doi.org/10.1103/PhysRevLett.124.171103

68. C. Caprini, R. Durrer, T. Konstandin, G. Servant, Phys. Rev. D 79, 083519 (2009). https://doi.org/10.1103/PhysRevD.79.083519

69. P. Niksa, M. Schlederer, G. Sigl, Class. Quantum Gravity 35(14), 144001 (2018). https://doi.org/10.1088/1361-6382/aac89c

70. L.P. Grishchuk, Phys. Usp. 48, 1235 (2005). https://doi.org/10. 1070/PU2005v048n12ABEH005795

71. F.A. Jenet, G.B. Hobbs, W. van Straten, R.N. Manchester, M. Bailes, J.P.W. Verbiest, R.T. Edwards, A.W. Hotan, J.M. Sarkissian, S.M. Ord, Astrophys. J. 653, 1571 (2006). https://doi.org/10.1086/ 508702

72. C. Caprini et al., JCAP 03, 024 (2020). https://doi.org/10.1088/ $1475-7516 / 2020 / 03 / 024$ 